\title{
COMPORTAMIENTO TÉRMICO Y EFICIENCIA ENERGÉTICA DE VIVIENDAS SOCIALES DE MADERA CON MUROS VENTILADOS
}

\author{
Marcelo González R ${ }^{1}$, Gonzalo Hernández $C^{2}$.
}

\section{RESUMEN}

Durante el año 2011 se encuentra en desarrollo un proyecto que contempla la construcción de 55 viviendas sociales con estructura de madera en la localidad de Arauco, Región del Bio Bio. Este proyecto contempló en el diseño de las viviendas la incorporación de muros con cámara ventilada.

Mediante cálculos térmicos basados en la normativa actual existente, se obtuvieron los flujos y pérdidas térmicas, y la eficiencia energética de la vivienda social de madera con muros ventilados; comparándola con una vivienda de madera sin muros ventilados y una vivienda con muros de ladrillo, todas de idéntica arquitectura.

El muro de madera con cámara ventilada presenta un flujo de calor menor que el muro de ladrillo, siendo esta reducción cercana al 70\%. En tanto, la diferencia de flujos térmicos entre el muro de madera con cámara ventilada y el muro de madera sin cámara ventilada es de sólo un $12 \%$.

Las viviendas de madera con muros ventilados y sin muros ventilados presentan un ahorro en el consumo de energía en calefacción significativo respecto a la vivienda de ladrillo, de un $48 \%$ y $45 \%$ respectivamente. Lo anterior se traduce en una disminución del consumo de combustible, menor contaminación intradomiciliaria y un ahorro económico para los habitantes.

\section{SUMMARY}

During the year 2011 is developing a project that includes the construction of 55 housing with a wooden structure in the town of Arauco, Region of Bio Bio. This project included the design of homes incorporating walls with ventilated chamber.

By thermal calculations based on current existing regulations were obtained flows and thermal losses, and energy efficiency of social wooden housing with walls ventilated, comparing it to a wooden house without ventilated walls and a housing with brick walls, all of the same architecture. 
The wooden wall with ventilated chamber has a lower heat flux than the brick wall, this reduction being about $70 \%$. Meanwhile, the difference of heat flows between the wooden wall with ventilated air and the wooden wall without ventilated air is only $12 \%$.

The wooden houses with ventilated walls and without ventilated walls have a significant savings in heating energy consumption to housing made of brick, $48 \%$ and $45 \%$ respectively. This results in a decrease in fuel consumption, reduced indoor air pollution and saving money for residents.

\section{INTRODUCCIÓN}

En la localidad de Arauco, región del Bío Bío, se encuentra en ejecución un proyecto que contempla la construcción de 55 viviendas sociales con estructura de madera. Este proyecto habitacional es subsidiado por el Gobierno de Chile como parte del proceso de reconstrucción después del terremoto del año 2010, y es ejecutado por la empresa Constructora Vega e Iglesias Ltda.

Para apoyar la construcción de las viviendas sociales de madera se suscribió un acuerdo entre el Instituto Forestal y la Comisión Escolar de la Capital de Quebec, permitiendo el trabajo de 14 Carpinteros de la Escuela de Oficios y Ocupaciones de la Industria de la Construcción de Quebec e incorporando en las 55 soluciones habitacionales muros con cámara ventilada, que mejoran su eficiencia térmica y generan un ahorro significativo para sus ocupantes por concepto de calefacción.

Este artículo pretende cuantificar el comportamiento térmico y la eficiencia energética de la vivienda social de madera con muros ventilados, comparándola con una vivienda de madera sin muros ventilados y una vivienda con muros de ladrillo, todas de idéntica arquitectura.

\section{MATERIALES Y MÉTODOS}

Las viviendas utilizadas en este estudio corresponden a un proyecto de 55 viviendas sociales de madera, de $52 \mathrm{~m}^{2}$ en un piso, emplazado en la localidad de Arauco,Región del Bio Bio, Chile.

Los cálculos térmicos se realizaron según las especificaciones de la norma chilena NCh 853 "Acondicionamiento térmico - Envolvente térmica de edificios - Cálculo de resistencias y transmitancias térmicas" (INN, 2003).

El cálculo de los coeficientes volumétricos globales de pérdidas térmicas (factores Gv1 y Gv2), se realizó según la norma NCh 1960: "Aislación térmica - Cálculo de coeficientes volumétricos globales de pérdidas térmicas" (INN, 1989).

Las conductividades térmicas de los materiales que conforman la envolvente de las viviendas, se obtuvieron de los valores que aparecen en el Manual de Aplicación de la 
Reglamentación Térmica (MART) y en la norma chilena NCh 853 (INN, 2003).

La Energía Requerida en Calefacción se obtuvo en base a los datos de Grados-días Anuales para la Localidad de Concepción (Sarmiento, 2007), tomando una temperatura base al interior de las viviendas de $18,1^{\circ} \mathrm{C}$. Se consideró además como período de calefacción los meses de abril a agosto, con 8 horas diarias de calefacción.

La descripción térmica de la envolvente y las superficies de los recintos de la vivienda de estudio se obtuvieron de los planos y especificaciones técnicas proporcionadas por la Constructora Vega e Iglesias.

Se obtuvieron las pérdidas térmicas para la vivienda de madera con muros ventilados (1), la vivienda de madera sin cámara ventilada (2) y la vivienda con muros de ladrillo (3). Para los tres tipos de vivienda se utilizó la misma arquitectura del proyecto original, solo modificando la materialidad de los muros perimetrales.

En las Figuras $\mathrm{N}^{\circ} 1$ y $\mathrm{N}^{\circ} 2$ se muestra el detalle de los muros de madera con y $\sin$ cámara ventilada, respectivamente. El muro de ladrillo se consideró de $14 \mathrm{~cm}$ de espesor.
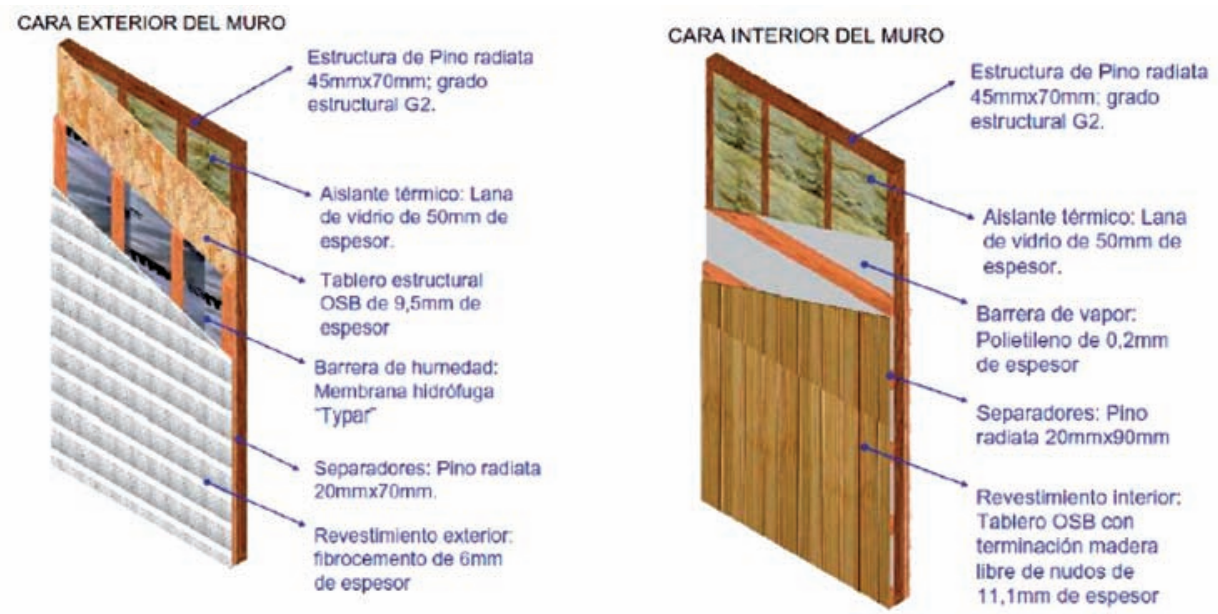

Figura $\mathrm{N}^{\circ} 1$

DETALLE DEL MURO DE MADERA CON CÁMARA VENTILADA 

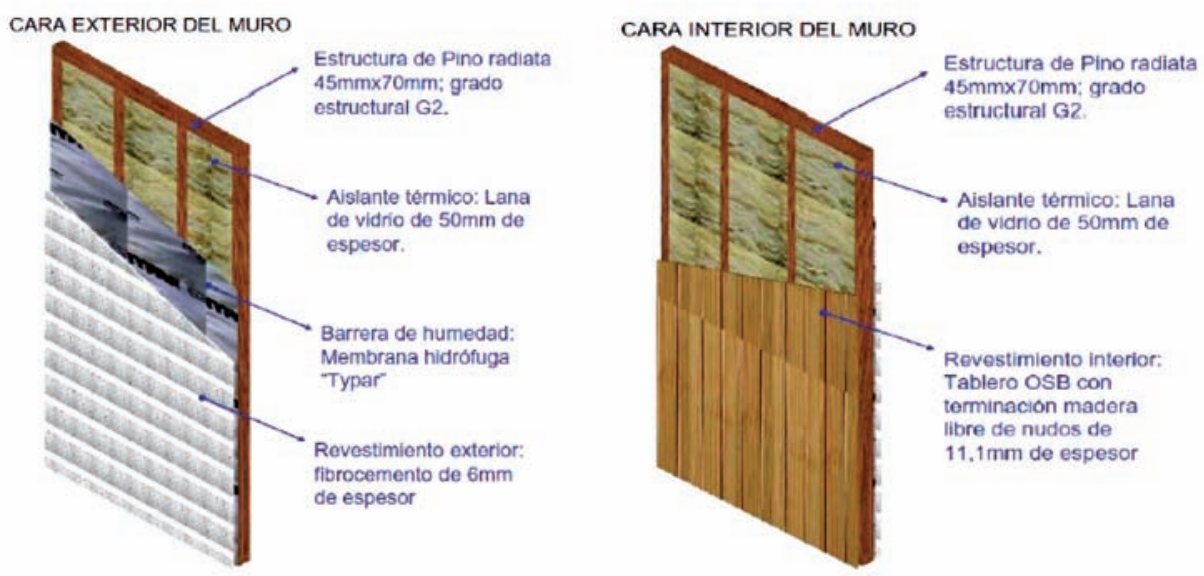

Figura $\mathrm{N}^{\circ} 2$

DETALLE DEL MURO DE MADERA SIN CÁMARA VENTILADA

\section{RESULTADOS}

\section{Transmitancias Térmicas}

La transmitancia térmica $(U)$ es el flujo de calor que pasa por unidad de superficie de un elemento y por grado de diferencia de temperatura entre los dos ambientes separados por dicho elemento (MINVU-IC, 2006).

Las transmitancias térmicas de los complejos de muros y techumbre, ventanas y puertas para los tres tipos de viviendas se pueden observar en los Cuadros $N^{\circ} 1, N^{\circ} 2$ y №3.

En la Figura № 3 se pueden apreciar las diferencias de flujos térmicos a través de los tres tipos de muro, donde el muro de ladrillo presenta una transmitancia térmica de 2,11 $\left[\mathrm{W} / \mathrm{m}^{\circ} \mathrm{C}\right]$, mientras que los muros de madera sin cámara ventilada y con cámara ventilada presentan transmitancias térmicas de $0,68\left[\mathrm{~W} / \mathrm{m}^{\circ} \mathrm{C}\right]$ y $0,60\left[\mathrm{~W} / \mathrm{m}^{\circ} \mathrm{C}\right]$, respectivamente. 


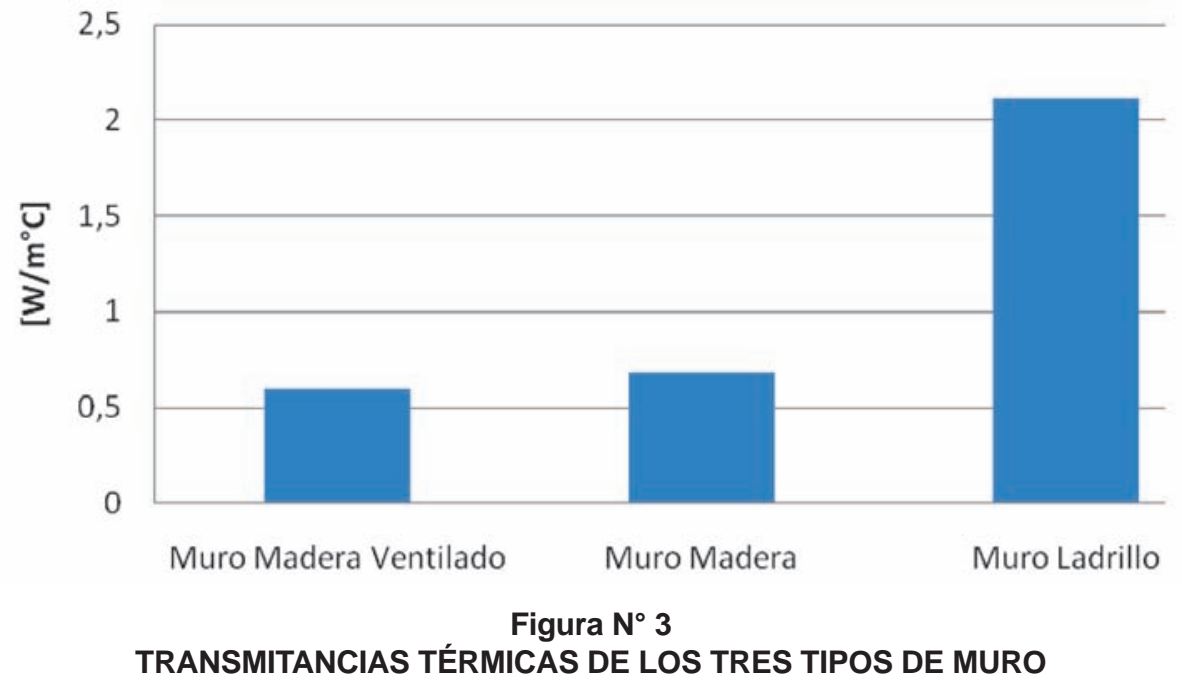

En base a los valores de transmitancia térmica de los muros, se tiene que el muro de madera con cámara ventilada reduce los flujos térmicos en un $72 \%$ respecto al muro de ladrillo, y en un $12 \%$ respecto al muro de madera sin cámara ventilada.

Es muy importante señalar que cuando menor sea la transmitancia térmica de un muro, menor será el riesgo de condensación de agua al interior de la vivienda, evitando de esta manera la proliferación de hongos y mohos que pueden causar enfermedades respiratorias en los habitantes (Rodriguez, 2007). Debido lo anterior, los muros de madera, con y sin cámara ventilada, presentan un riesgo de condensación de agua significativamente menor al de un muro de ladrillo.

Además, se debe destacar que para la condición de verano, la incorporación de muros ventilados en las viviendas permite evitar el sobrecalentamiento de la vivienda y mantener una temperatura de $3-4^{\circ} \mathrm{C}$ menor a la temperatura exterior (Martinez, 2010). 


\section{Cuadro $\mathrm{N}^{\circ} 1$}

\section{VIVIENDA DE MADERA CON MUROS VENTILADOS}

\begin{tabular}{|l|l|c|c|c|c|}
\hline \multicolumn{2}{|l|}{ Elementos de la envolvente } & $\begin{array}{c}\text { Superficie } \\
{[\mathbf{m} 2]}\end{array}$ & $\begin{array}{c}\mathbf{R t} \\
{\left[\mathbf{m} \mathbf{2}^{\circ} \mathbf{C} / \mathbf{W}\right]}\end{array}$ & $\begin{array}{c}\mathbf{U} \\
{\left[\mathbf{W} / \mathbf{m} \mathbf{}^{\circ} \mathrm{C}\right]}\end{array}$ & $\begin{array}{c}\text { U ponderado } \\
{\left[\mathbf{W} / \mathbf{m}^{\circ} \mathbf{C}\right]}\end{array}$ \\
\hline Muros & Muros zona seca & 57,55 & 1,68 & 0,59 & \multirow{2}{*}{0,60} \\
\hline & Muros zona humeda & 5,72 & 1,62 & 0,62 & \multirow{2}{*}{0,39} \\
\hline Techumbre & Techumbre zona seca & 44,18 & 2,59 & 0,39 & \multirow{2}{*}{5,77} \\
\hline & Techumbre zona húmeda & 7,82 & 2,58 & 0,39 & \multicolumn{2}{|c|}{2,31} \\
\hline Ventanas & & 7,04 & 0,17 & 0,43 & \multicolumn{2}{|c|}{} \\
\hline
\end{tabular}

Cuadro $\mathrm{N}^{\circ} 2$

VIVIENDA DE MADERA SIN MUROS VENTILADOS

\begin{tabular}{|l|l|c|c|c|c|}
\hline \multicolumn{2}{|l|}{ Elementos de la envolvente } & $\begin{array}{c}\text { Superficie } \\
{[\mathbf{m} 2]}\end{array}$ & $\begin{array}{c}\mathbf{R t} \\
{\left[\mathbf{m} \mathbf{2}^{\circ} \mathbf{C} / \mathbf{W}\right]}\end{array}$ & $\begin{array}{c}\mathbf{U} \\
{\left[\mathbf{W} / \mathbf{m} \mathbf{2}^{\circ} \mathbf{C}\right]}\end{array}$ & $\begin{array}{c}\text { U ponderado } \\
{\left[\mathbf{W} / \mathbf{m}^{\circ} \mathbf{C}\right]}\end{array}$ \\
\hline Muros & Muros zona seca & 57,55 & 1,49 & 0,67 & \multirow{2}{*}{0,68} \\
\hline & Muros zona húmeda & 5,72 & 1,42 & 0,70 & \multirow{2}{*}{0,39} \\
\hline Techumbre & Techumbre zona seca & 44,18 & 2,59 & 0,39 & 0,39 \\
\hline & Techumbre zona húmeda & 7,82 & 2,58 & \multicolumn{2}{|c|}{5,77} \\
\hline Ventanas & & 7,04 & 0,17 & \multicolumn{2}{|c|}{2,31} \\
\hline
\end{tabular}

\section{Cuadro $\mathrm{N}^{\circ} 3$ \\ VIVIENDA CON MUROS DE LADRILLO}

\begin{tabular}{|l|l|c|c|c|c|}
\hline \multicolumn{2}{|l|}{ Elementos de la envolvente } & $\begin{array}{c}\text { Superficie } \\
{[\mathbf{m} 2]}\end{array}$ & $\begin{array}{c}\mathbf{R t} \\
{\left[\mathbf{m}{ }^{\circ} \mathbf{C} / \mathbf{W}\right]}\end{array}$ & $\begin{array}{c}\mathbf{U} \\
{\left[\mathbf{W} / \mathbf{m} \mathbf{2}^{\circ} \mathbf{C}\right]}\end{array}$ & $\begin{array}{c}\text { U ponderado [W/ } \\
\left.\mathbf{m}^{\circ} \mathbf{C}\right]\end{array}$ \\
\hline Muros & Muros zona seca & 57,55 & 0,47 & 2,11 & \multirow{2}{*}{2,11} \\
\hline & Muros zona humeda & 5,72 & 0,47 & 2,11 & \multirow{2}{*}{0,39} \\
\hline Techumbre & Techumbre zona seca & 44,18 & 2,59 & 0,39 & \\
\hline & Techumbre zona húmeda & 7,82 & 2,58 & 0,39 & \multicolumn{2}{|c|}{5,77} \\
\hline Ventanas & 7,04 & 0,17 & \multicolumn{2}{|c|}{2,31} \\
\hline Puertas & 3,00 & 0,43 & & \multicolumn{2}{|c|}{} \\
\hline
\end{tabular}




\section{Pérdidas Térmicas Globales y Eficiencia Energética}

Los factores globales de perdidas térmicas calculados, fueron el factor Gv1, que permite obtener la razón entre los flujos térmicos a través de la envolvente de la vivienda y el producto de su volumen interior y la diferencia de temperatura entre el interior y el exterior; y el factor Gv2 que es similar al anterior pero agregando las pérdidas térmicas por ventilación e infiltración de aire (INN, 1989). En el Cuadro $\mathrm{N}^{\circ} 4$ se presenta los valores globales de pérdidas térmicas para los tres tipos de vivienda.

Basado en las diferencias de las pérdidas globales totales (Gv2) entre los tres tipos de vivienda se obtiene que:

- $\quad$ El ahorro en el consumo de energía en calefacción de la vivienda de madera con muros ventilados, es de un $48 \%$ respecto de la vivienda con muros de ladrillo.

- El ahorro en el consumo de energía en calefacción de la vivienda de madera sin muros ventilados, es de un $45 \%$ respecto de la vivienda con muros de ladrillo.

- El ahorro en el consumo de energía en calefacción de la vivienda de madera con muros ventilados, es de un $5 \%$ respecto de la vivienda de madera sin muros ventilados.

\section{Cuadro $\mathrm{N}^{\circ} 4$ \\ FACTORES GLOBALES DE PÉRDIDAS TÉRMICAS DE LOS TRES TIPOS DE VIVIENDA}

\begin{tabular}{|l|c|c|}
\hline Vivienda & $\begin{array}{c}\text { Gv1 } \\
{\left[\mathbf{W} / \mathbf{m}^{3 \circ} \mathbf{C}\right]}\end{array}$ & $\begin{array}{c}\mathbf{G v 2} \\
{\left[\mathbf{W} / \mathbf{m}^{3 \circ} \mathbf{C}\right]}\end{array}$ \\
\hline Madera muros ventilados & 0,85 & 1,38 \\
Madera & 0,89 & 1,42 \\
Albañilería ladríllo & 1,62 & 2,15 \\
\hline
\end{tabular}

\section{Energía Requerida en Calefacción (ERC)}

La energía requerida en calefacción que se obtuvo se observa en el Cuadro $N^{\circ} 5$. Para las viviendas de madera con y sin muro ventilado la ERC es cercana a los $25 \mathrm{~kW}$-h/ $\mathrm{m}^{2} \mathrm{año}$; mientras que para la vivienda de ladrillo el consumo energético asciende a los 46,4 $\mathrm{kW}-\mathrm{h} / \mathrm{m}^{2} a \tilde{n} o$.

Para las viviendas en estudio, una familia que habita la vivienda de ladrillo deberá gastar cerca de $\$ 179.000$ en calefacción durante el invierno, mientras que la que habita en la vivienda de madera con muros ventilados gastará aproximadamente $\$ 94.000^{1}$. El ahorro

1 Considerando parafina (kerosene doméstico) como combustible, a un precio de mercado de $\$ 700$ por litro, y utilizando los valores del Cuadro $\mathrm{N}^{\circ} 6$. 
económico en calefacción es de \$85.000 por año.

Además del ahorro económico producido, la disminución de combustible (gas licuado, parafina y leña), permitirá disminuir la contaminación intradomiciliaria afectando positivamente la salud de los habitantes y su calidad de vida (Caceres et al., 2001)

\section{Cuadro $\mathrm{N}^{\circ} 5$ \\ ENERGÍA REQUERIDA EN CALEFACCIÓN PARA LOS TRES TIPOS DE VIVIENDA}

\begin{tabular}{|l|c|c|}
\hline Vivienda & $\begin{array}{c}\text { ERC } \\
\text { [kW-h/m2año] }\end{array}$ & $\begin{array}{c}\text { ERC } \\
{[\mathbf{k W}-\mathbf{h} / \mathbf{a n ̃ o}]}\end{array}$ \\
\hline Madera muros ventilados & 24,30 & 1263,62 \\
\hline Madera & 25,46 & 1324,02 \\
\hline Albañilería ladríllo & 46,38 & 2411,69 \\
\hline
\end{tabular}

\section{Cuadro $\mathrm{N}^{\circ} 6$}

COMBUSTIBLE REQUERIDO EN CALEFACCIÓN PARA LOS TRES TIPOS DE VIVIENDA

\begin{tabular}{|l|c|c|c|c|}
\hline Vivienda & $\begin{array}{c}\text { Leña } \\
\text { [kg/año] }\end{array}$ & $\begin{array}{c}\text { Parafina } \\
\text { [l/año] }\end{array}$ & $\begin{array}{c}\text { Gas licuado [kg/ } \\
\text { año] }\end{array}$ & $\begin{array}{c}\text { Electricidad } \\
\text { [kW-h/año] }\end{array}$ \\
\hline Madera muros ventilados & 1034,96 & 134,30 & 94,54 & 1263,62 \\
\hline Madera & 1084,43 & 140,72 & 99,06 & 1324,02 \\
\hline Albañilería ladríllo & 1975,29 & 256,31 & 180,43 & 2411,69 \\
\hline
\end{tabular}

\section{CONCLUSIONES}

El muro de madera con cámara ventilada presenta un flujo de calor menor que el muro de ladrillo, siendo esta reducción cercana al 70\%. En tanto, la diferencia de flujos térmicos entre el muro de madera con cámara ventilada y el muro de madera sin cámara ventilada es de sólo un $12 \%$.

Las diferencias en los flujos térmicos de los muros, se traducen en diferencias de pérdidas térmicas en los tres tipos de vivienda, donde las viviendas de madera, con y sin muros ventilados, respecto de la vivienda de ladrillo, presentan un ahorro significativo en el consumo de energía para calefacción, de un 48\% y 45\% respectivamente.

La eficiencia energética de las viviendas de madera respecto a la vivienda de ladrillo, conlleva una disminución del consumo de combustible y la contaminación intradomiciliaria, además de un ahorro económico para los habitantes. 


\section{REFERENCIAS}

Caceres, D.,Adonis, M. y Retamal,C., 2001. Contaminaciónintradomiciliaria enunsectorde extrema pobreza de la comuna de La Pintana. Rev. méd. Chile. [En línea]. <http://www.scielo. cl/scielo.php?script=sci_arttext\&pid=S0034-98872001000100005\&lng=es\&nrm=iso $>$. ISSN 0034-9887. [Consulta: 12 de Agosto de 2011]

INN, 1989. Norma chilena oficial; NCh 1960.Of89: Aislación térmica-Cálculo de coeficientes volumétricos globales de pérdidas térmicas. Instituto Nacional de Normalización, Chile.

INN, 2003. Norma chilena oficial; NCh 853.Of2003: Acondicionamiento térmico Envolvente térmica de edificios - Cálculo de resistencias y transmitancias térmicas. Instituto Nacional de Normalización, Chile.

Martínez, P., 2010. Diseño envolvente: Una Tecnología disponible para la reconstrucción en madera. Seminario Reconstruyamos a Chile en Madera, Facultad de Ciencias Forestales, Universidad de Talca.

MINVU-IC, 2006. Manual de aplicación reglamentación térmica: Ordenanza General de Urbanismo y construcciones artículo 4.1.10. Primera edición. Editorial Edicolor. Santiago, Chile. 54 p. Ministerio de Vivienda y Urbanismo; Instituto de la Construcción..

Rodríguez, G., 2007. Clima y construcción habitacional: Conceptos térmicos. Apuntes del curso Física de la Construcción. Facultad de Ciencias Físicas y Matemáticas, Universidad de Chile.

Sarmiento, P., 2007. Energía Solar en Arquitectura y Construcción. Primera edición. Editorial Ril. Santiago, Chile. 343 p. 
